\title{
Parallel Algorithms for Collaborative Filtering
}

\author{
Edward Y. Chang ${ }^{1,2}$ \\ ${ }^{1}$ Google Beijing Research, Beijing 100084, China \\ ${ }^{2}$ University of California, Santa Barbara, CA 93106, USA \\ edchang@google.com
}

Collaborative filtering has been widely used to predict the interests of a user. Given a users past activities, collaborative filtering predicts the users future preferences. This talk presents techniques and discoveries of our recent parallelization effort on collaborative filtering algorithms. In particular, parallel association mining and parallel latent Dirichlet allocation will be presented and their pros and cons analyzed. Some counter-intuitive results will also be presented to stimulate future parallel optimization research. 